\title{
Color analysis of saffron (Crocus sativus L.) as potential natural colorant
}

\begin{abstract}
Purpose

The purpose of the paper is to study the effect of color stability on introducing chenodeoxycholic acid (CDCA) into a colored liquid extract from saffron and determine the color quality of the extract over a nine-month period.

Design/methodology/approach

Six colored liquid samples with different CDCA contents ranging from 0 to $45 \mathrm{Wt} \% \%$ have been successfully prepared. Chromaticity ( $\left.\mathrm{C}^{*}\right)$, color saturation (s), UV-Vis spectroscopy and coloring strength studies have been assessed to determine how CDCA influences the color properties and to study the color quality over time. The color quality was analyzed using the Commission Internationale de l'Eclairage (CIE) system.
\end{abstract}

Findings

All results obtained revealed that the addition of CDCA significantly influenced the overall color performance of the saffron extraction. However, the most pronounced improvement was recorded with the use of $45 \mathrm{Wt} . \% \mathrm{CDCA}$. The sample exhibited the highest color quality at the end of nine months of storage with highest absorbance: $C^{*}$ value $=91.38$, color saturation $=$ 0.96 and coloring strength $=687$.

Practical implications

This preliminary study offers significant findings for further research focused on stability of natural colorants extracted from Spanish saffron that can provide benefits for future applications especially in coating industry, food, agriculture, medicine and others.

Originality/value

The values of this work can be observed from the information and evidence provided by CIE color stability in terms of chromaticity and saturation, as well as UV-Vis spectrophotometric measurement. It showed that the addition of CDCA additive can help to prolong and enhance the natural colorant properties from Spanish saffron (Crocus sativus L.) for nine month of storage. This proved that by adding additives such as CDCA the saffron colorant can be maintained. To the best of the authors' concern, this is the first time CDCA is used to prevent color degradation of natural colorant from saffron.

Keyword: Extraction; Saffron; Color measurement; Crocus sativus L.; Chenodeoxycholic acid 